\title{
Observation of nonlinear variations in a three-vertex geometric phase in a two-photon polarization qutrit
}

\section{$\operatorname{AUTHOR}(\mathrm{S})$ :}

Ogawa, Kazuhisa; Tamate, Shuhei; Kobayashi, Hirokazu; Nakanishi, Toshihiro; Kitano, Masao

\section{CITATION:}

Ogawa, Kazuhisa ...[et al]. Observation of nonlinear variations in a three-vertex geometric phase in a two-photon polarization qutrit. Physical Review A 2015, 91(6): 062118.

\section{ISSUE DATE:}

2015-06-16

URL:

http://hdl.handle.net/2433/201997

RIGHT:

(c) 2015 American Physical Society 
PHYSICAL REVIEW A 91, 062118 (2015)

\title{
Observation of nonlinear variations in a three-vertex geometric phase in a two-photon polarization qutrit
}

\author{
Kazuhisa Ogawa, ${ }^{1, *}$ Shuhei Tamate, ${ }^{2}$ Hirokazu Kobayashi, ${ }^{3}$ Toshihiro Nakanishi, ${ }^{1}$ and Masao Kitano ${ }^{1}$ \\ ${ }^{1}$ Department of Electronic Science and Engineering, Kyoto University, Kyoto 615-8510, Japan \\ ${ }^{2}$ National Institute of Informatics, Hitotsubashi, Chiyoda-ku, Tokyo 101-8430, Japan \\ ${ }^{3}$ Department of Electronic and Photonic System Engineering, Kochi University of Technology, Tosayamada-cho, Kochi 782-8502, Japan
}

(Received 8 April 2015; published 16 June 2015)

\begin{abstract}
We observed experimentally nonlinear variations in the three-vertex geometric phase in a two-photon polarization qutrit. The three-vertex geometric phase is defined by three quantum states, which generally form a three-state (qutrit) system. By changing one of the three constituent states, we observed two rapid increases in the three-vertex geometric phase. The observed variations are inherent in a three-state system and cannot be observed in a two-state system. We used a time-reversed two-photon interferometer to measure the geometric phase with much more intense signals than those of a typical two-photon interferometer.
\end{abstract}

DOI: 10.1103/PhysRevA.91.062118

PACS number(s): 03.65.Vf, 03.65.Ta, 42.65.-k

\section{INTRODUCTION}

The geometric phase is a fundamental concept in many areas of physics. It was discovered by Berry [1] as an additional phase factor that emerges in adiabatic and cyclic evolution of a quantum state. The definition of the geometric phase was extended to the nonadiabatic [2] and noncyclic [3] cases and was finally generalized on the basis of kinematic ideas by Mukunda and Simon [4]. In their formulation, the geometric phase is defined by a trajectory on the quantum state space and is represented as a sum of the following three-vertex geometric phases:

$$
\gamma\left(\psi_{1}, \psi_{2}, \psi_{3}\right):=\arg \left\langle\psi_{1} \mid \psi_{3}\right\rangle\left\langle\psi_{3} \mid \psi_{2}\right\rangle\left\langle\psi_{2} \mid \psi_{1}\right\rangle,
$$

which is defined by three quantum states [5]. Therefore, the three-vertex geometric phase is regarded as a fundamental building block of an arbitrary geometric phase.

The three-vertex geometric phase is ubiquitous in various physical systems involving three different states. In optical systems, the three-vertex geometric phase appears in an additional phase factor after three polarization projections [5] or three reflections [6] and in the interference patterns of three differently polarized beams [7]. In the problem distinguishing three quantum states, the three-vertex geometric phase is an important factor characterizing their distinguishability [810]. In addition, the quantum eraser [11] and weak-value amplification [12] are related to the three-vertex geometric phase defined by the initial, intermediate, and final states in the systems $[13,14]$.

The three-vertex geometric phase has been widely studied in a two-state (qubit) system. In a two-state system, the threevertex geometric phase is geometrically represented as the area of a spherical triangle formed by the three constituent states on the Bloch (Poincaré) sphere [5]. Various nonlinear behaviors of the three-vertex geometric phase in a two-state system have been investigated using the Bloch sphere representation and observed in several optical experiments [13,15-18].

However, the three arbitrary states that define a three-vertex geometric phase generally span a three-dimensional Hilbert

\footnotetext{
*ogawa@giga.kuee.kyoto-u.ac.jp
}

space; therefore, we need to treat a three-state (qutrit) system to investigate the general properties of the three-vertex geometric phase. In our previous study [19], we constructed a geometric representation of the three-vertex geometric phase in a threestate system on the Bloch sphere. Using the Bloch sphere representation, we predicted some nonlinear variations in the three-vertex geometric phase inherent in a three-state system.

In this paper we experimentally observe the nonlinear variations in the three-vertex geometric phase inherent in a three-state system with an optical interferometer. We employ the polarizations of two photons in the same spatiotemporal mode (a two-photon polarization qutrit) as a three-state system [20-23]. In our setup, the three-vertex geometric phase exhibits two rapid increases with respect to a change in one of the three constituent states. We use a time-reversed two-photon interferometer [24] for the measurement. Unlike the typical method of measuring the geometric phase in two-photon polarization [25-27], our setup can obtain vastly more intense signals and can be implemented using classical light.

This paper is organized as follows. In Sec. II we briefly review the theory of the three-vertex geometric phase in a three-state system investigated in Ref. [19]. In Sec. III we describe the experimental observation of the nonlinear variations in the three-vertex geometric phase. We also discuss the advantages of using a time-reversed two-photon interferometer for the experiments. Finally, we summarize the findings of our study in Sec. IV.

\section{THEORY}

We describe the Bloch sphere representation of the threevertex geometric phase in a three-state system [19]. We also derive the nonlinear variations in the three-vertex geometric phase inherent in a three-state system, which are experimentally observed in Sec. III.

A three-state system can be identified in terms of a symmetrized two-qubit system. The symmetrized two-qubit state $|\Psi\rangle$ is described as

$$
|\Psi\rangle=k\left(|\psi\rangle\left|\psi^{\prime}\right\rangle+\left|\psi^{\prime}\right\rangle|\psi\rangle\right),
$$

where $|\psi\rangle$ and $\left|\psi^{\prime}\right\rangle$ are qubit states and $k$ is a normalization factor (in what follows, we omit $k$ for simplicity). Here $|\Psi\rangle$ can 
(a)

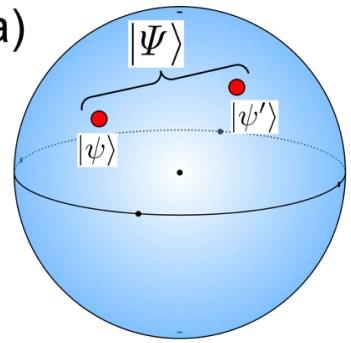

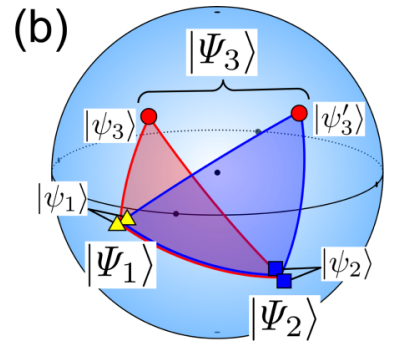

FIG. 1. (Color online) (a) Bloch sphere representation of the symmetrized two-qubit state $|\Psi\rangle$. (b) Bloch sphere representation of the three-vertex geometric phase of the standard triplet $\gamma\left(\Psi_{1}, \Psi_{2}, \Psi_{3}\right)$, which is proportional to the sum of the area of the two spherical triangles.

be uniquely depicted as the two points corresponding to $|\psi\rangle$ and $\left|\psi^{\prime}\right\rangle$ on the Bloch sphere (Majorana's stellar representation [28-31]), as shown in Fig. 1(a).

To visualize the three-vertex geometric phase on the Bloch sphere, we consider the following standard triplet:

$$
\begin{aligned}
& \left|\Psi_{1}\right\rangle=\left|\psi_{1}\right\rangle\left|\psi_{1}\right\rangle, \quad\left|\Psi_{2}\right\rangle=\left|\psi_{2}\right\rangle\left|\psi_{2}\right\rangle, \\
& \left|\Psi_{3}\right\rangle=\left|\psi_{3}\right\rangle\left|\psi_{3}^{\prime}\right\rangle+\left|\psi_{3}^{\prime}\right\rangle\left|\psi_{3}\right\rangle,
\end{aligned}
$$

where $\left|\Psi_{1}\right\rangle$ and $\left|\Psi_{2}\right\rangle$ are product states and $\left|\Psi_{3}\right\rangle$ is an arbitrary symmetrized two-qubit state. Although the standard triplet is a special set of three states, any set of three states can be mapped onto a standard triplet by applying the proper unitary transformation [19]. The three-vertex geometric phase of the standard triplet is expressed as the sum of two three-vertex geometric phases in two-state systems:

$$
\gamma\left(\Psi_{1}, \Psi_{2}, \Psi_{3}\right)=\gamma\left(\psi_{1}, \psi_{2}, \psi_{3}\right)+\gamma\left(\psi_{1}, \psi_{2}, \psi_{3}^{\prime}\right) .
$$

Because a three-vertex geometric phase in a two-state system is equal to $-1 / 2$ times the area of a spherical triangle on the Bloch sphere [5], the three-vertex geometric phase of the standard triplet can be depicted as the area of two spherical triangles on the Bloch sphere, as shown in Fig. 1(b). In this manner, we can represent an arbitrary three-vertex geometric phase in a three-state system on the Bloch sphere.

We next derive the nonlinear variations in the threevertex geometric phase inherent in a three-state system from the Bloch sphere representation. We employ a two-photon polarization qutrit as a three-state system. Here $|H\rangle$ and $|V\rangle$ denote the horizontal and vertical polarization states, respectively.

We now consider the following standard triplet of twophoton polarization qutrits:

$$
\begin{gathered}
\left|\Psi_{1}\right\rangle=\left|\psi_{1}\right\rangle\left|\psi_{1}\right\rangle, \quad\left|\Psi_{2}\right\rangle=\left|\psi_{2}\right\rangle\left|\psi_{2}\right\rangle, \\
\left|\Psi_{3}(\phi)\right\rangle=\left|\psi_{3}(\phi)\right\rangle\left|\psi_{3}^{\prime}(\phi)\right\rangle+\left|\psi_{3}^{\prime}(\phi)\right\rangle\left|\psi_{3}(\phi)\right\rangle,
\end{gathered}
$$

where

$$
\begin{gathered}
\left|\psi_{1}\right\rangle:=\cos (\theta / 2)|H\rangle+i \sin (\theta / 2)|V\rangle, \\
\left|\psi_{2}\right\rangle:=\cos (\theta / 2)|H\rangle-i \sin (\theta / 2)|V\rangle,
\end{gathered}
$$
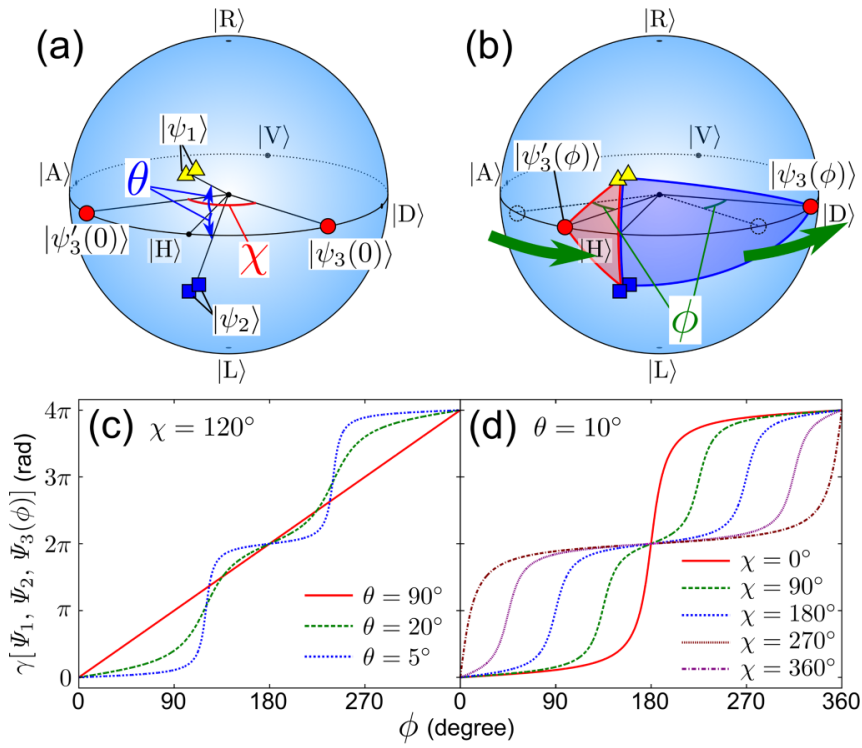

FIG. 2. (Color online) (a) Bloch sphere representation of the states given by Eqs. (7)-(10) when $\phi=0^{\circ}$. Here $\theta$ is the half angle between the states $\left|\psi_{1}\right\rangle$ and $\left|\psi_{2}\right\rangle$ and $\chi$ is the angle between the states $\left|\psi_{3}(\phi)\right\rangle$ and $\left|\psi_{3}^{\prime}(\phi)\right\rangle$. (b) Bloch sphere representation of the geometric phase $\gamma\left[\Psi_{1}, \Psi_{2}, \Psi_{3}(\phi)\right]$ when the two red circles $\left|\psi_{3}(\phi)\right\rangle$ and $\left|\psi_{3}^{\prime}(\phi)\right\rangle$ are rotated along the equator. When the red circles pass through the reverse side of the Bloch sphere, the area of the spherical triangles increases rapidly. In addition, as the angle between the yellow triangles and the blue squares decreases, the area of the two spherical triangles increases more rapidly. Also shown are the variations in $\gamma\left[\Psi_{1}, \Psi_{2}, \Psi_{3}(\phi)\right]$ with respect to $\phi$ (c) for several values of $\theta$ when $\chi=120^{\circ}$ and (d) for several values of $\chi$ when $\theta=10^{\circ}$.

$$
\begin{aligned}
\left|\psi_{3}(\phi)\right\rangle & :=\cos \left(\frac{\chi}{4}+\frac{\phi}{2}\right)|H\rangle+\sin \left(\frac{\chi}{4}+\frac{\phi}{2}\right)|V\rangle, \\
\left|\psi_{3}^{\prime}(\phi)\right\rangle & :=\cos \left(\frac{\chi}{4}-\frac{\phi}{2}\right)|H\rangle-\sin \left(\frac{\chi}{4}-\frac{\phi}{2}\right)|V\rangle .
\end{aligned}
$$

This standard triplet is depicted in Fig. 2(a). The parameters $\theta$ and $\chi$ are fixed at certain values. We change $\phi$ to rotate the two red circles $\left|\psi_{3}(\phi)\right\rangle$ and $\left|\psi_{3}^{\prime}(\phi)\right\rangle$ along the equator on the Bloch sphere, as shown in Fig. 2(b). The three-vertex geometric phase $\gamma\left[\Psi_{1}, \Psi_{2}, \Psi_{3}(\phi)\right]$ is calculated as

$$
\begin{aligned}
& \gamma\left[\Psi_{1}, \Psi_{2}, \Psi_{3}(\phi)\right]=\gamma\left[\psi_{1}, \psi_{2}, \psi_{3}(\phi)\right]+\gamma\left[\psi_{1}, \psi_{2}, \psi_{3}^{\prime}(\phi)\right], \\
& \gamma\left[\psi_{1}, \psi_{2}, \psi_{3}(\phi)\right]=-2 \tan ^{-1}\left[\tan \frac{\theta}{2} \tan \left(\frac{\chi}{4}+\frac{\phi}{2}\right)\right] \\
& \gamma\left[\psi_{1}, \psi_{2}, \psi_{3}^{\prime}(\phi)\right]=2 \tan ^{-1}\left[\tan \frac{\theta}{2} \tan \left(\frac{\chi}{4}-\frac{\phi}{2}\right)\right] .
\end{aligned}
$$

The variations in $\gamma\left[\Psi_{1}, \Psi_{2}, \Psi_{3}(\phi)\right]$ with respect to $\phi$ for several values of $\theta$ and $\chi$ are shown in Figs. 2(c) and 2(d). These figures indicate that the variations in $\gamma\left[\Psi_{1}, \Psi_{2}, \Psi_{3}(\phi)\right]$ exhibit two rapid increases by $2 \pi$ at the angles $\phi=180^{\circ} \pm \chi / 2$ and as the angle $\theta$ decreases, the geometric phase increases more rapidly. These rapid variations in $\gamma\left[\Psi_{1}, \Psi_{2}, \Psi_{3}(\phi)\right]$ are 
interpreted as nonlinear variations in the area of the two spherical triangles on the Bloch sphere.

\section{EXPERIMENT}

We next describe our experimental observation of the nonlinear variations in the three-vertex geometric phase in a three-state system derived in Sec. II. In Sec. III A we describe our experimental setup for measuring the geometric phase using an optical interferometer. In Sec. III B we show the measured nonlinear variations in the geometric phase. In Sec. III C we discuss the advantages of our experimental setup for measuring the geometric phase in two-photon polarization.

\section{A. Experimental setup}

In the experiment, we measure the three-vertex geometric phase using a quantum eraser [13,25]. Let us consider the interferometer of a photon pair shown in Fig. 3(a). The input photon pair with the initial two-photon polarization qutrit state $\left|\Psi_{0}\right\rangle$ is first split into two arms by a beam splitter. The twophoton polarization qutrit states of the upper and lower mode are transformed into $\left|\Psi_{1}\right\rangle$ and $\left|\Psi_{2}\right\rangle$, respectively, by unitary operations (e.g., half- or quarter-wave plates). Subsequently, both of the two-photon polarization qutrit states are projected onto $\left|\Psi_{3}\right\rangle$ and the two path modes are combined by another beam splitter. By changing the relative phase $\delta$ between the two path modes, we can observe interference fringes. When the final state $\left|\Psi_{3}\right\rangle$ varies, the variation in the three-vertex geometric phase $\gamma\left(\Psi_{1}, \Psi_{2}, \Psi_{3}\right)$ can be measured from a phase shift of the interference fringes, as shown in Fig. 3(b).

The actual experimental setup is shown in Fig. 3(c), which implements the measurement method using a quantum eraser. We used a femtosecond fiber laser (center wavelength $782 \mathrm{~nm}$, pulse duration $74.5 \mathrm{fs}$, average power $54 \mathrm{~mW}$, repetition rate $100 \mathrm{MHz}$ ) to create transform-limited pulsed light with horizontal polarization. The input pulse enters the preparation section, which forms an unbalanced Michelson interferometer including three quarter-wave plates (QWPs). The optical path difference $x$ between the two arms of the interferometer can be changed by a piezoelectric actuator and is adjusted to about $100 \mu \mathrm{m}$. The two output pulses of the interferometer are substantially separated in time and hardly interfere with each other. After passing through the third QWP, the two-photon polarization qutrit states of the later and earlier pulses are transformed into $\left|\Psi_{1}\right\rangle=\left|\psi_{1}\right\rangle\left|\psi_{1}\right\rangle$ and $\left|\Psi_{2}\right\rangle=\left|\psi_{2}\right\rangle\left|\psi_{2}\right\rangle$ in Eq. (5), respectively.

The pulses next pass through the projection section, which consists of three half-wave plates (HWPs), polarizing and nonpolarizing beam splitters (PBS and BS), and a 1-mm-long $\beta$-barium borate (BBO) crystal for collinear type-II sumfrequency generation (SFG). The HWPs, PBS, and BS convert the polarizations $\left|\psi_{3}(\phi)\right\rangle$ and $\left|\psi_{3}^{\prime}(\phi)\right\rangle$ in Eqs. (9) and (10) into $|H\rangle$ and $|V\rangle$, respectively. Subsequently, the BBO crystal converts only two photons with the two-photon polarization qutrit state $|H\rangle|V\rangle+|V\rangle|H\rangle$ into a sum-frequency photon. Therefore, the entire section projects the two-photon polarization qutrit state onto $\left|\Psi_{3}(\phi)\right\rangle$ in Eq. (6).

The two sum-frequency pulses interfere with each other with respect to each frequency component as shown in

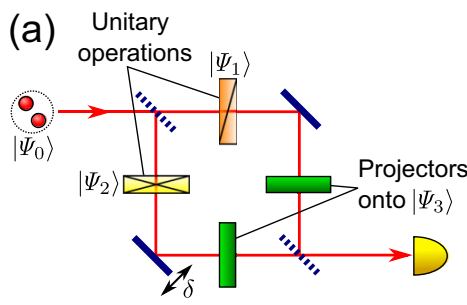

(b) Variation of geometric phase

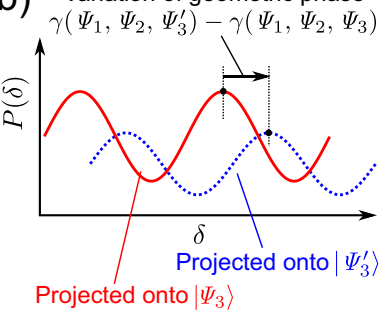

(c)

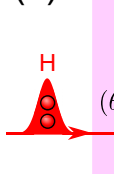

Preparation section
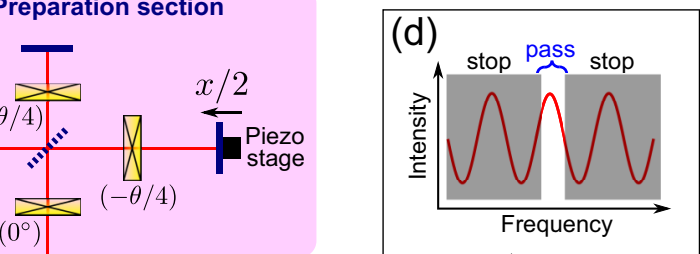

$\left(0^{\circ}\right)$
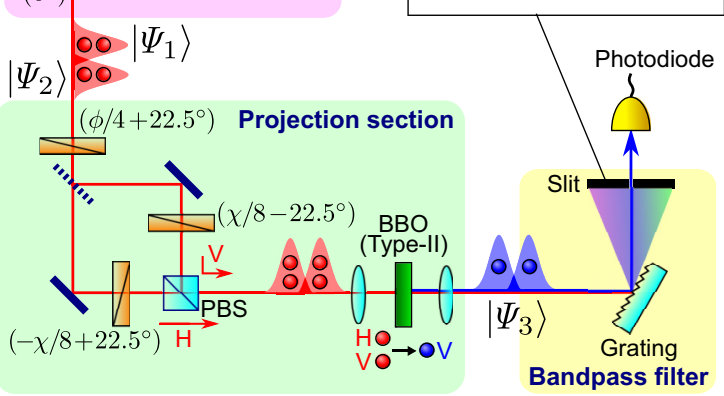

Mirror
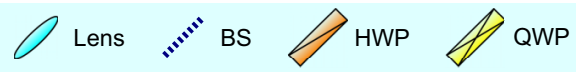

FIG. 3. (Color online) (a) Schematic illustration of the setup for measuring the three-vertex geometric phase in a quantum eraser. (b) Projection probability $P(\delta)$ for different final internal states $\left|\Psi_{3}\right\rangle$ and $\left|\Psi_{3}^{\prime}\right\rangle$. From the phase shift of the interference fringes, we can measure the variation in the three-vertex geometric phase $\gamma\left(\Psi_{1}, \Psi_{2}, \Psi_{3}^{\prime}\right)-$ $\gamma\left(\Psi_{1}, \Psi_{2}, \Psi_{3}\right)$. (c) Experimental setup for measuring the three-vertex geometric phase in the two-photon polarization qutrit: QWP, quarterwave plate; HWP, half-wave plate; BS, (nonpolarizing) beam splitter; PBS, polarizing beam splitter; $\mathrm{BBO}, \beta$-barium borate crystal. The values in parentheses next to the QWPs and HWPs denote the angles of their fast axes from the horizontal axis. The parameter $\theta$ is adjusted by changing the angles of the QWPs. The parameters $\chi$ and $\phi$ are adjusted by changing the angles of the HWPs. (d) Spectral intensity of the light after the BBO crystal. When the optical path difference $x$ is changed, the distribution shifts transversely. By extracting the light in a narrow frequency region, we can observe the interference fringes with high visibility.

Fig. 3(d). We filter the two pulses to pass a 0.23-nm bandwidth centered at around $391 \mathrm{~nm}$ by a 1200-line/mm aluminumcoated diffraction grating followed by a slit. The extracted two pulses produce interference with high visibility (the detailed calculation is provided in the Appendix). The optical power is measured by a Si photodiode (New Focus, Model 2151). We measured the interference fringes as a function of the optical path difference $x$ for various values of $\theta, \chi$, and $\phi$ and derived the variations in the three-vertex geometric phase from the shifts of the fringes. 

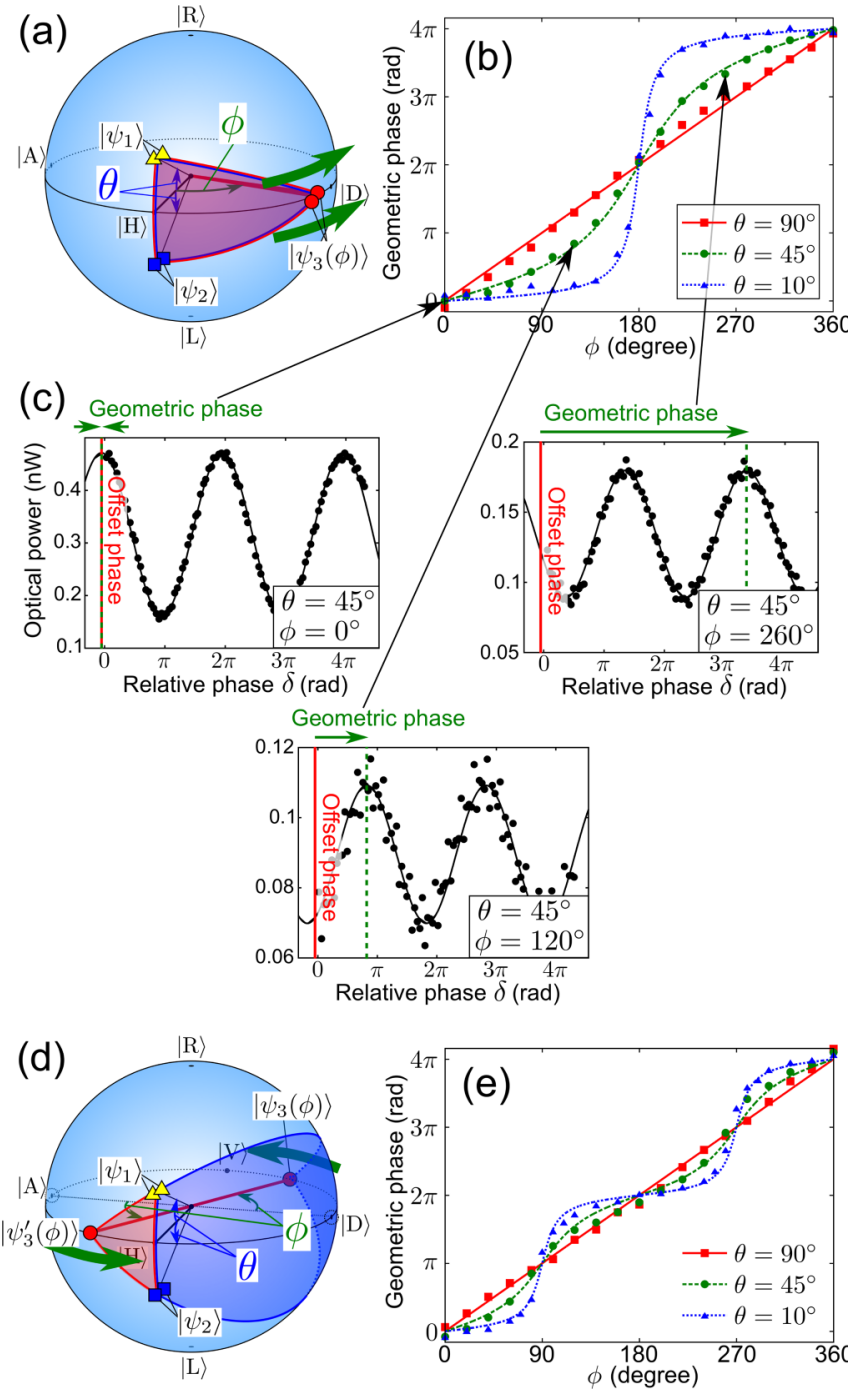

FIG. 4. (Color online) Variations in the three-vertex geometric phase for (a) - (c) $\chi=0^{\circ}$ and (d) and (e) $\chi=180^{\circ}$. (a) and (d) Bloch sphere representation of the three-vertex geometric phase. (b) and (e) Measured variations in the geometric phase with respect to $\phi$. (c) Some instances of the measured interference fringes for several values of $\phi$ when $\theta=45^{\circ}$. As the Bloch sphere representation predicts, the geometric phase increases rapidly by (b) $4 \pi$ at $\phi=180^{\circ}$ and (e) $2 \pi$ at $\phi=90^{\circ}$ and $270^{\circ}$. We can see that as the angle $\theta$ decreases, the geometric phase increases more rapidly.

\section{B. Results}

We first measured the variations in the three-vertex geometric phase with respect to $\phi$ for several values of $\theta$ when $\chi=0^{\circ}$ [Figs. 4(a)-4(c)] and $\chi=180^{\circ}$ [Figs. 4(d) and 4(e)]. From the Bloch sphere representation [Figs. 4(a) and 4(d)], we can predict that the geometric phase increases rapidly by $4 \pi$ at $\phi=180^{\circ}$ when $\chi=0^{\circ}$ and by $2 \pi$ at $\phi=90^{\circ}$ and $270^{\circ}$ when $\chi=180^{\circ}$. Figures 4(b) and 4(e) show the measurement results, where the dots and lines denote the measurement data and the theoretical lines, respectively. Figure 4(c) shows some instances of the measured interference fringes for several values of $\phi$ when $\theta=45^{\circ}$. By comparing the phase shifts among these fringes, we measured the relative variation in the three-vertex geometric phase with respect to a certain offset phase. We determined the offset phase by fitting the measurement data of the relative variation in the three-vertex geometric phase to the theoretical lines. The offset phase was determined for every setting of the parameters $\theta$ and $\chi$. The measurement results agree well with the theoretical prediction. As the angle $\theta$ decreases, the geometric phase increases more rapidly.

We also measured the variations in the three-vertex geometric phase with respect to $\phi$ for several values of $\chi$ when $\theta=10^{\circ}$. From the Bloch sphere representation (the upper panels in Fig. 5), we can predict that the two values of $\phi$ at which the geometric phase increases rapidly depend on $\chi: \phi=180^{\circ} \pm \chi / 2$. The graphs in the lower panels in Fig. 5 show the measurement results, where the dots and lines denote the measurement data and the theoretical lines, respectively, and we determined the offset phase of the measurement data in the same manner as described above. We can see that the measurement results agree well with the theoretical prediction by our Bloch sphere representation and the locations of the two jumping points depend on $\chi$.

\section{Discussion}

Here we note the advantages of using our experimental setup for measuring the geometric phase in two-photon polarization for the purpose of this study. Such measurements have typically been made using a two-photon interferometer [25-27]. In the two-photon interferometer, we need to generate photon pairs by spontaneous parametric down-conversion (SPDC) and to detect them by coincidence counting. Because of the low efficiency of generation and detection of entangled photon pairs, the two-photon interferometer often suffers from weak output signals, which makes our estimation of the geometric phase uncertain.

In contrast, we employ a time-reversed two-photon interferometer [24] for measuring the geometric phase. The timereversed two-photon interferometer up-converts two photons into a sum-frequency photon by SFG instead of generating photon pairs by SPDC. Because the output power of SFG is proportional to the square of the input power, we can observe vastly more intense interference signals. Moreover, coincidence counting of photon pairs is not needed in the time-reversed two-photon interferometer; therefore, we can measure the geometric phase in two-photon polarization using a simpler setup. Indeed, this technique cannot be used for observing the nonclassical effect of photon pairs [25,27] because the technique is implemented using classical light. However, for the purpose of this study, that is, to verify that the geometric phases in a two-photon polarization qutrit change as predicted by the Bloch sphere representation, this technique is more advantageous than the conventional method using a two-photon interferometer.

In our experiment, the output signal power is minimized when $\theta=10^{\circ}, \chi=0^{\circ}$, and $\phi=180^{\circ}$. Even in this condition, the measured average power of the interference fringes is 1.1 $\mathrm{pW}$, which corresponds to $2.1 \times 10^{6}$ photons/s. This output power is three orders of magnitude greater than that in previous experiments [25-27]. 

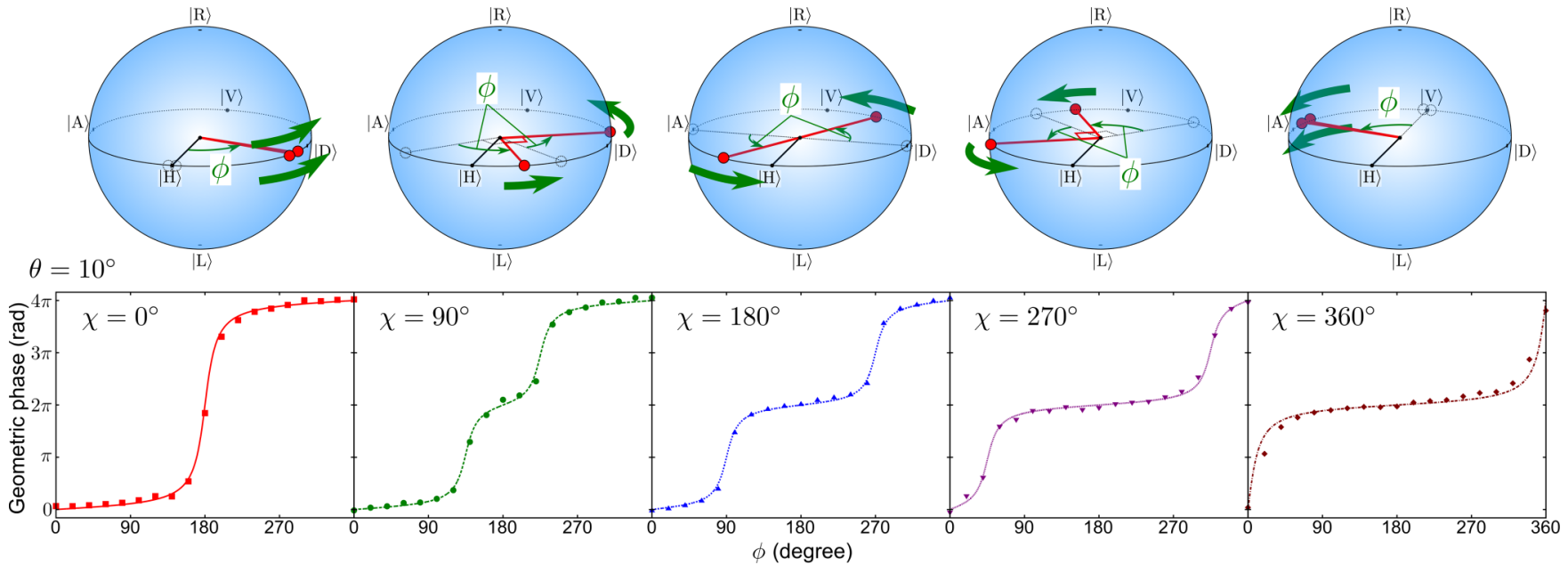

FIG. 5. (Color online) Variations in the three-vertex geometric phase with respect to $\phi$ for several values of $\chi$ when $\theta=10^{\circ}$. The upper panels are the Bloch sphere representations of $\left|\psi_{3}(\phi)\right\rangle$ and $\left|\psi_{3}^{\prime}(\phi)\right\rangle$. The graphs in the lower panels show the measurement results corresponding to each Bloch sphere. As the Bloch sphere representation predicts, the locations of the two jumping points of the geometric phase depend on $\chi$.

\section{CONCLUSION}

We presented optical experiments for measuring the threevertex geometric phase in a two-photon polarization qutrit. We experimentally demonstrated the nonlinear variations in the three-vertex geometric phase, which is inherent in a three-state system such as a two-photon polarization qutrit. The nonlinear variations are predicted by the Bloch sphere representation and all the measurement results agree well with the theoretical prediction. We also noted that our measurement method for the geometric phase using a time-reversed two-photon interferometer enables us to obtain vastly more intense output signals. This measurement method can be used for highintensity measurement of other properties of the geometric phase. We expect that the Bloch sphere representation of the geometric phase in high-dimensional systems and the measurement technique shown in this paper will motivate the investigation of a new nonlinear variation of the geometric phase in higher-dimensional systems and will enable new quantum optical technology using the geometric phase.

\section{ACKNOWLEDGMENTS}

This research was supported by Japan Society for the Promotion of Science KAKENHI Grants No. 22109004 and No. 25287101.

\section{APPENDIX: CALCULATION OF TIME-REVERSED TWO-PHOTON INTERFEROMETER}

We show the calculation of the time-reversed two-photon interferometer [24] used in our experiments. In the calculation, we omit the polarization degree of freedom.

We describe the complex electric-field amplitude of the input light as $E(\omega):=\exp \left[-\left(\omega-\omega_{0}\right)^{2} / 2 \Delta \omega^{2}\right]$, where $\omega_{0}$ and $\Delta \omega$ are the central frequency and the standard deviation of the frequency bandwidth, respectively. The field amplitude after passing through the unbalanced Michelson interferometer is given by $E(\omega)\left(1+e^{i \omega x / c}\right) / 2$, where $x$ is the optical path difference between the two arms of the interferometer and $c$ is the speed of light. The BBO crystal for SFG converts the field amplitude into the following convolution integral $E_{\mathrm{SFG}}(\omega)$ :

$$
\begin{aligned}
E_{\mathrm{SFG}}(\omega) \propto & \int_{-\infty}^{\infty} d \omega^{\prime} E\left(\omega^{\prime}\right)\left[1+e^{i \omega^{\prime} x / c}\right] E\left(\omega-\omega^{\prime}\right) \\
& \times\left[1+e^{i\left(\omega-\omega^{\prime}\right) x / c}\right] \\
= & \int_{-\infty}^{\infty} d \omega^{\prime} E\left(\omega^{\prime}\right) E\left(\omega-\omega^{\prime}\right) \\
& \times\left[1+e^{i \omega^{\prime} x / c}+e^{i\left(\omega-\omega^{\prime}\right) x / c}+e^{i \omega x / c}\right] .
\end{aligned}
$$

When $x$ is large enough, the integrals of the second and third terms are negligibly small. The integrals of the first and forth terms are calculated as

$$
E_{\mathrm{SFG}}(\omega) \propto \exp \frac{-\left(\omega-2 \omega_{0}\right)^{2}}{4 \Delta \omega^{2}}\left(1+e^{i \omega x / c}\right) .
$$

This equation means that the frequency components that undergo constructive interference are converted into sumfrequency light effectively compared with those that undergo destructive interference. To observe the interference fringes with high visibility, the bandpass filter composed of the grating and the slit extracts a narrow frequency range around $2 \omega_{0}$. If the transmission spectrum of the bandpass filter is sufficiently narrow, the measured intensity $I(x)$ after the bandpass filter is given by

$$
I(x)=\left|E\left(2 \omega_{0}\right)\right|^{2} \propto 1+\cos \left(2 \omega_{0} x / c\right) .
$$

Therefore, we can observe the two-photon phase superresolution by using the time-reversed two-photon interferometer.
[1] M. V. Berry, Proc. R. Soc. London Ser. A 392, 45 (1984).

[2] Y. Aharonov and J. Anandan, Phys. Rev. Lett. 58, 1593 (1987).
[3] J. Samuel and R. Bhandari, Phys. Rev. Lett. 60, 2339 (1988).

[4] N. Mukunda and R. Simon, Ann. Phys. (N.Y.) 228, 205 (1993). 
[5] S. Pancharatnam, Proc. Indian Acad. Sci. A 44, 247 (1956).

[6] M. Kitano, T. Yabuzaki, and T. Ogawa, Phys. Rev. Lett. 58, 523 (1987).

[7] H. Kobayashi, S. Tamate, T. Nakanishi, K. Sugiyama, and M. Kitano, Phys. Rev. A 81, 012104 (2010).

[8] R. Jozsa and J. Schlienz, Phys. Rev. A 62, 012301 (2000).

[9] J. A. Bergou, U. Futschik, and E. Feldman, Phys. Rev. Lett. 108, 250502 (2012).

[10] H. Sugimoto, T. Hashimoto, M. Horibe, and A. Hayashi, Phys. Rev. A 82, 032338 (2010).

[11] M. O. Scully and K. Drühl, Phys. Rev. A 25, 2208 (1982).

[12] Y. Aharonov, D. Z. Albert, and L. Vaidman, Phys. Rev. Lett. 60, 1351 (1988).

[13] H. Kobayashi, S. Tamate, T. Nakanishi, K. Sugiyama, and M. Kitano, J. Phys. Soc. Jpn. 80, 034401 (2011).

[14] S. Tamate, H. Kobayashi, T. Nakanishi, K. Sugiyama, and M. Kitano, New J. Phys. 11, 093025 (2009).

[15] H. Schmitzer, S. Klein, and W. Dultz, Phys. Rev. Lett. 71, 1530 (1993).

[16] S. P. Tewari, V. Ashoka, and M. Sree Ramana, Opt. Commun. 120, 235 (1995).

[17] Q. Li, L. Gong, Y. Gao, and Y. Chen, Opt. Commun. 169, 17 (1999).

[18] B. Hils, W. Dultz, and W. Martienssen, Phys. Rev. E 60, 2322 (1999).
[19] S. Tamate, K. Ogawa, and M. Kitano, Phys. Rev. A 84, 052114 (2011).

[20] Y. I. Bogdanov, M. V. Chekhova, S. P. Kulik, G. A. Maslennikov, A. A. Zhukov, C. H. Oh, and M. K. Tey, Phys. Rev. Lett. 93, 230503 (2004).

[21] B. P. Lanyon, T. J. Weinhold, N. K. Langford, J. L. O’Brien, K. J. Resch, A. Gilchrist, and A. G. White, Phys. Rev. Lett. 100, 060504 (2008).

[22] G. M. D'Ariano, P. Mataloni, and M. F. Sacchi, Phys. Rev. A 71, 062337 (2005).

[23] G. Vallone, E. Pomarico, F. De Martini, P. Mataloni, and M. Barbieri, Phys. Rev. A 76, 012319 (2007).

[24] K. Ogawa, S. Tamate, H. Kobayashi, T. Nakanishi, and M. Kitano, Phys. Rev. A 88, 063813 (2013).

[25] H. Kobayashi, Y. Ikeda, S. Tamate, T. Nakanishi, and M. Kitano, Phys. Rev. A 83, 063808 (2011).

[26] J. Brendel, W. Dultz, and W. Martienssen, Phys. Rev. A 52, 2551 (1995).

[27] J. C. Loredo, M. A. Broome, D. H. Smith, and A. G. White, Phys. Rev. Lett. 112, 143603 (2014).

[28] E. Majorana, Nuovo Cimento 9, 43 (1932).

[29] H. D. Liu and L. B. Fu, Phys. Rev. Lett. 113, 240403 (2014).

[30] P. Bruno, Phys. Rev. Lett. 108, 240402 (2012).

[31] J. Hannay, J. Phys. A 31, L53 (1998). 